\title{
Perspectives for heavy-flavour measurements in ALICE with the upgraded Inner Tracking System
}

\author{
F. Fionda ${ }^{1, a}$ on behalf of the ALICE Collaboration \\ ${ }^{1}$ University and INFN - Bari, Italy
}

\begin{abstract}
The ALICE detector at the LHC is specifically designed to investigate the properties of the hot and dense medium, consisting of deconfined quarks and gluons (QGP), created in high energy heavy-ion collisions. Heavy-flavour particles are wellsuited probes to investigate the properties of such a medium, since they are mainly produced in the initial phase and they subsequently experience the full evolution of the collision. In the ALICE experiment, the Inner Tracking System (ITS) plays an important role for heavy-flavour measurements. Interesting results have been obtained by analyzing $\mathrm{pp}, \mathrm{p}-\mathrm{Pb}$ and $\mathrm{Pb}-\mathrm{Pb}$ data collected so far. The upgrade of the ITS, combined with the expected increase of LHC luminosity in $\mathrm{Pb}-\mathrm{Pb}$ collisions, will allow the current performance for heavy flavour measurements to be significantly improved in particular in the low momentum region, and new measurements will become accessible (e.g. the $\Lambda_{\mathrm{c}}$ baryon in $\mathrm{Pb}-\mathrm{Pb}$ collisions). The perspectives for heavy-flavour measurements expected after the second long shut-down (LS2) of LHC will be discussed.
\end{abstract}

\section{Physics motivations for studying heavy-flavour}

In ultra-relativistic heavy-ion collisions the QGP can be studied by means of various probes emerging from the collision; in particular "rare" probes like charm and beauty hadrons allow for a detailed characterization of the formation and the evolution of the QGP state. Heavy quarks are predominantly produced in the initial hard nucleon-nucleon scatterings. The subsequent interaction with the colored partonic plasma constituents, via both elastic (collisional energy loss) [1] and inelastic (mediuminduced gluon radiation, or radiative energy loss) [2] processes, leads to a substantial energy loss and, possibly, to the thermalization of low transverse momentum $\left(p_{\mathrm{T}}\right)$ heavy quarks. Because of the smaller color coupling factor, quarks should lose less energy than gluons. In addition, the "dead-cone effect" [3], which reduces small-angle gluon radiation for heavy quarks with moderate energy-over-mass value, is expected to determine a smaller energy loss for heavy quarks with respect to light ones. The effects of energy loss can be quantified using the nuclear modification factor $R_{\mathrm{AA}}$, which is defined as the ratio of the particle yield in heavy-ion collisions compared to that in pp, scaled by the number of binary nucleon-nucleon collisions $N_{\text {coll }}$. In particular, it is interesting to compare among them and with theoretical predictions the nuclear modification factors of several hadron species, containing charm, beauty and light quarks.

\footnotetext{
a e-mail: fiorella.fionda@ba.infn.it
} 
Further insight into the interaction mechanisms of heavy quarks with the medium can be achieved by measuring the "elliptic flow" of heavy-flavour hadrons in non-central heavy-ion collisions. The elliptic flow is defined as the second order coefficient $\left(v_{2}\right)$ in the Fourier expansion of the azimuthal distribution of produced particles. If heavy quarks thermalize in the medium or if they interact strongly with it, the heavy-flavour hadrons produced by heavy quark hadronization should inherit the medium azimuthal anisotropy, resulting in a non-zero $v_{2}$.

Heavy quark hadronization in the presence of the QGP can be studied through the production measurements of strange-charmed meson $\mathrm{D}_{\mathrm{s}}$. It is expected [4] that in a deconfined state the abundances of parton species should quickly reach their equilibrium values, resulting in a strangeness enhancement in heavy-ion collisions with respect to pp collisions. Then if the recombination takes part dominantly to the hadronization process [5], a larger production of $D_{\mathrm{s}}$ mesons, and consequently an higher $R_{\mathrm{AA}}$, should be observed with respect to non-strange D-mesons. Finally the baryon-overmeson ratio for charm $\left(\Lambda_{c} / D\right)$ and beauty $\left(\Lambda_{b} / B\right)$ hadrons observed in heavy-ion collisions with respect to pp gives additional information about the role of the recombination mechanism in heavy quark hadronization.

\section{Heavy-flavour measurements in ALICE}

ALICE [6] is the dedicated heavy-ion experiment at the LHC. It consists of a central barrel detector, covering the pseudorapidity interval $|\eta|<0.9$, placed inside a large solenoidal magnet providing a 0.5 $\mathrm{T}$ field, and a dimuon spectrometer located at forward rapidity $(-4<\eta<-2.5)$.

In the central barrel the Inner Tracking System (ITS) and the Time Projection Chamber (TPC) provide high precision tracking of charged particles, and in particular the ITS is crucial for the reconstruction of "displaced" vertices of heavy-flavour particle decays. It consist of six cylindrical layers employing three different silicon detector technologies: pixels for the two innermost layers, drift detectors for the two intermediate layers and strips for the two outermost layers. Concerning the Particle IDentification (PID), hadrons are identified by the energy loss measurements in the TPC and information from the Time Of Flight (TOF). Electrons are also identified with TPC and TOF and at higher momentum by using the ElectroMagnetic Calorimeter (EMCal) and the Transition Radiation Detector (TRD).

Several measurements in the heavy-flavour sector have been performed by analyzing $\mathrm{pp}, \mathrm{Pb}-\mathrm{Pb}$ and $\mathrm{p}-\mathrm{Pb}$ data collected during the LHC Run I (2009-2013). The main feature of the ALICE detector is the possibility to allow these measurements down to low $p_{\mathrm{T}}$. Currently in $\mathrm{Pb}-\mathrm{Pb}$ collisions the precision of the heavy-flavour measurements is limited due to the large combinatorial background. Since any trigger scheme is not applicable or inefficient at low $p_{\mathrm{T}}$ a further improvement for these measurements can be obtained by: (i) increasing the collected statistics; (ii) improving significantly the vertexing and tracking resolution in order to optimize the background rejection based on topological selections. The latter point is mandatory in some cases, as for the reconstruction of the $\Lambda_{c}$ baryon. This represents the most challenging heavy-flavour measurement, because of its small mean proper decay length $(c \tau=59 \mu \mathrm{m})$. ALICE is therefore preparing a major upgrade of its detectors that will be installed during the second Long LHC Shutdown (LS2) in the years 2018-2019. The main features of the ALICE upgrade strategy, and in particular for the new Inner Tracking System, will be described in the next sections. 


\section{The ALICE upgrade program}

The ALICE upgrade plans are discussed in the ALICE Upgrade Letter of Intent [7]. An important part of this program is represented by a significantly improved readout capability to record $\mathrm{Pb}-\mathrm{Pb}$ collisions at an interaction rate of about $50 \mathrm{kHz}$, which corresponds to the instantaneous luminosity of $6 \times 10^{27} \mathrm{~cm}^{-2} \mathrm{~s}^{-1}$, foreseen after the LS2. The main points of the ALICE upgrade strategy are summarized below:

- installation of a thinner beam pipe with a smaller diameter (from the present value of $29.8 \mathrm{~mm}$ down to $20.0 \mathrm{~mm}$ );

- installation of a new Inner Tracking System, described in the next section;

- upgrade of the Time-Projection-Chamber, in particular the replacement of Multi-Wire-ProportionalChambers (MWPCs) with micro-pattern gaseous detectors and the upgrade of read-out electronics;

- upgrade of the readout electronics of the Transition Radiation Detector, the Time-Of-Flight, the Photon Spectrometer, the Electromagnetic Calorimeter and the Muon Spectrometer;

- installation of a new Muon Forward Tracker (MFT), a telescope of silicon pixel detectors that will allow the secondary vertex reconstruction for heavy-flavour hadrons detected in the muon decay channels;

- upgrade of the forward trigger detectors and of the Zero Degree Calorimeter;

- upgrade of the DAQ system, the High Level Trigger and the Offline framework.

\section{The upgrade of the Inner Tracking System}

The detector layout, the technical implementations and the expected performance of the upgraded Inner Tracking System are described in the Technical Design Report (TDR) [8]. The new ITS design goals will improve the impact parameter resolution (by a factor 3 and 5 in $r \varphi$ and z, respectively), the tracking efficiency, the transverse momentum resolution and the readout capabilities.

In order to improve the impact parameter resolution, the first layer will be placed closer to the interaction point: the reduction of the beam pipe diameter in the center of ALICE will allow the installation of the first ITS layer down to $22 \mathrm{~mm}$ (the first layer of the current ITS is at $39 \mathrm{~mm}$ ). A significant improvement of impact parameter and momentum resolution, as well as of the tracking efficiency at low $p_{\mathrm{T}}$ will be obtained by reducing the total material budget per layer: thanks to the usage of Monolithic Active Pixel Sensors (MAPS) and the optimization of front-end electronics, it will be reduced from the present $1.14 \% X_{0}$ to $0.3 \% X_{0}$ for the three innermost layers and to $0.8 \% X_{0}$ for the outer layers. Both impact parameter and transverse momentum resolutions will be improved by increasing the detector segmentation: all layers will be segmented in pixels with dimensions $20 \times 30 \mu \mathrm{m}^{2}$ (to be compared with $50 \times 425 \mu \mathrm{m}^{2}$ for the current ITS). Finally the tracking efficiency and $p_{\mathrm{T}}$ resolution will be further improved by increasing up to seven the number of detector layers (six for the present ITS).

The new ITS will not measure the energy loss in the silicon layers since detailed studies have shown that the benefit would be marginal for the main items of the physics program with the upgraded detector. In addition, the measurement of the energy loss would require an increase of the material budget and of the readout time. 


\section{Expected performance for heavy-flavour measurements}

The expected performance for heavy-flavour measurements in $\mathrm{Pb}-\mathrm{Pb}$ collisions after the LS2 depends mainly on the significant improvement of the impact parameter and secondary vertex resolutions, as well as on the increase of the collected statistics. In particular, the goal is to collect a $\mathrm{Pb}-\mathrm{Pb}$ sample of $10 \mathrm{nb}^{-1}$ (corresponding to about $10^{11}$ interactions).
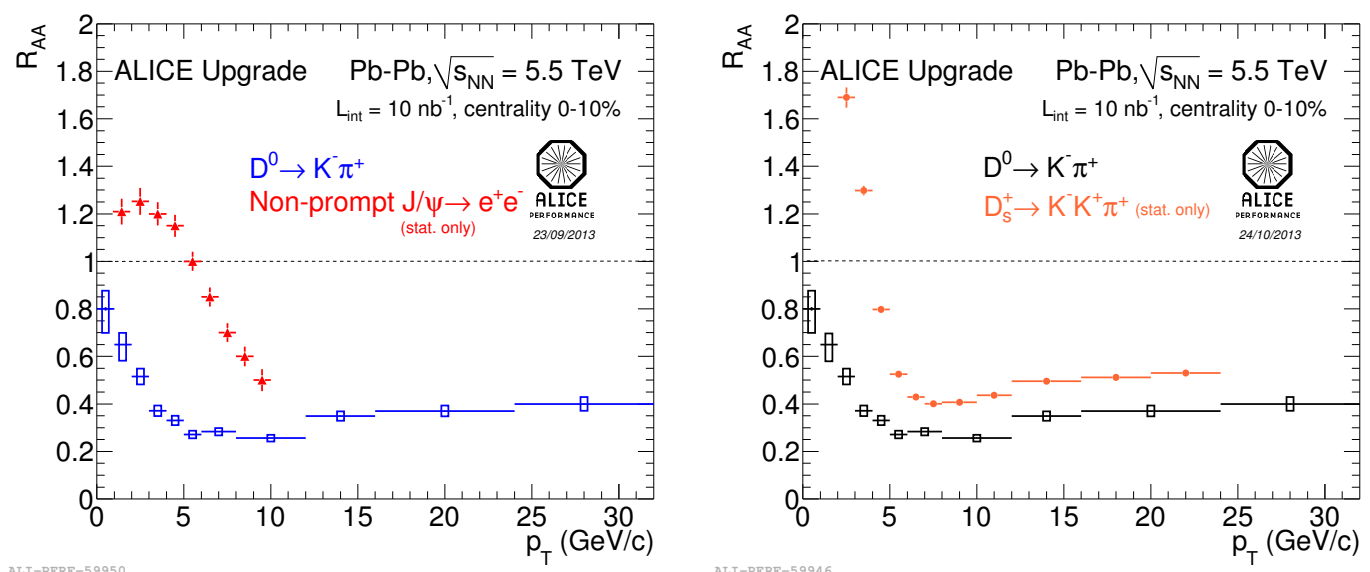

Figure 1. Nuclear modification factor of prompt $\mathrm{D}^{0}$ shown with $\mathrm{J} / \psi$ from beauty hadron decays (left-hand panel) and with $\mathrm{D}_{\mathrm{s}}$ (right-hand panel) for central $\mathrm{Pb}-\mathrm{Pb}$ collisions $\left(\mathrm{L}_{\mathrm{int}}=10 \mathrm{nb}^{-1}\right)$.
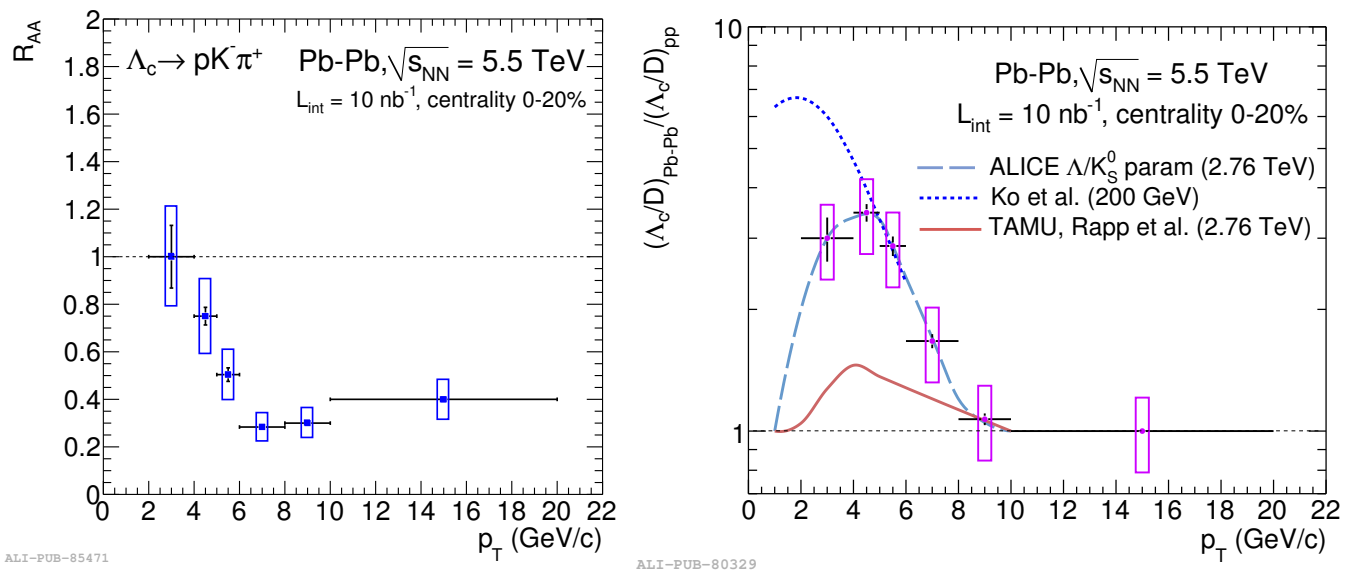

Figure 2. Left: nuclear modification factor of $\Lambda_{\mathrm{c}}$ for $\mathrm{Pb}-\mathrm{Pb}$ collisions in centrality $0-20 \%$. Right: double ratio $\left(\Lambda_{\mathrm{c}} / \mathrm{D}\right)$ for $\mathrm{Pb}-\mathrm{Pb}$ collisions in $0-10 \%$ w.r.t. pp.

The left-hand panel of Fig. 1 shows the nuclear modification factor of prompt $\mathrm{D}^{0}$ (both statistic and systematic uncertainties are shown) together with the one of $\mathrm{J} / \psi$ from beauty hadron decays (only statistical uncertainties shown) expected in central $\mathrm{Pb}-\mathrm{Pb}$ collisions $(0-10 \%)$. The precision of the measurements will give the possibility to study the mass-dependence of charm and beauty energy loss in the QGP in a wide transverse momentum range. In the right-hand panel of Fig. 1 
the same nuclear modification factor of prompt $\mathrm{D}^{0}$ is shown along with that of $\mathrm{D}_{\mathrm{s}}$ mesons. The small statistical uncertainties will enable a precise comparison of the suppression of strange and non-strange D-mesons.
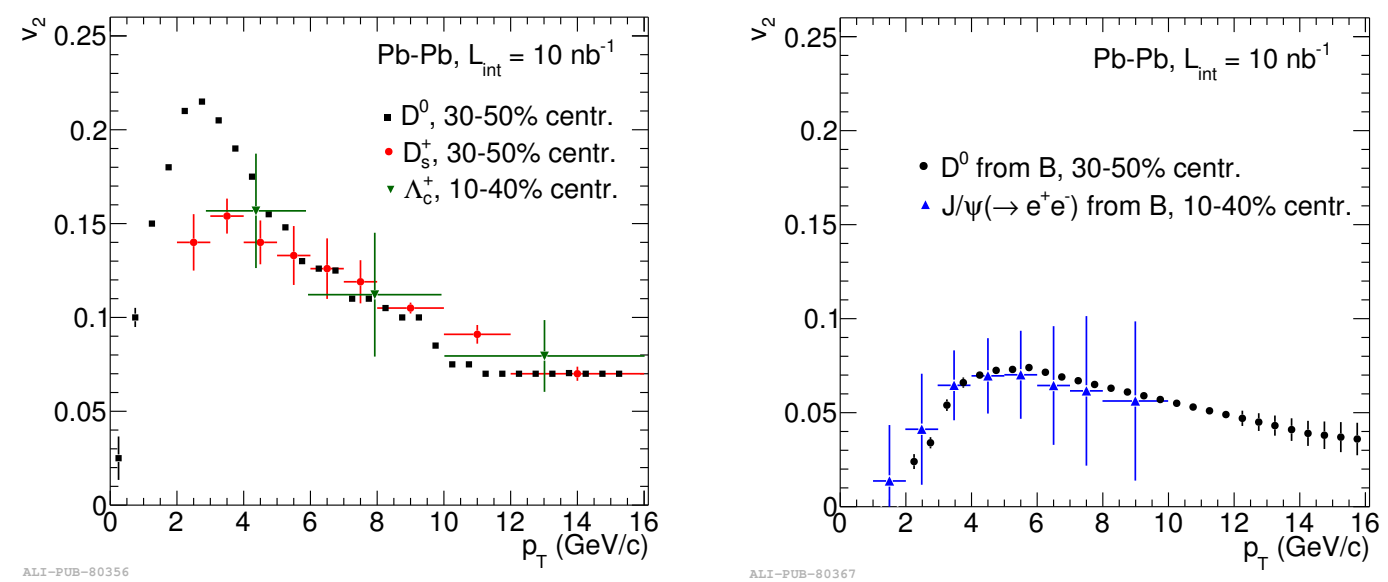

Figure 3. Elliptic flow of prompt $\mathrm{D}^{0}, \mathrm{D}_{\mathrm{s}}$ and $\Lambda_{\mathrm{c}}$ (left-hand panel) and of $\mathrm{D}^{0}$ and non-prompt $\mathrm{J} / \psi$ (right-hand panel) with estimated statistical uncertainties for $L_{\text {int }}=10 \mathrm{nb}^{-1}$ in semi-central $\mathrm{Pb}-\mathrm{Pb}$ collisions.

One of the benchmark measurements studied for the ALICE upgrade physics program is the $\Lambda_{\mathrm{c}}$ reconstruction. Because of its very small decay length, the current impact parameter resolution $(\sim 60 \mu \mathrm{m}$ at $p_{\mathrm{T}}=1 \mathrm{GeV} / c$ ) is not enough for observing the $\Lambda_{\mathrm{c}}$ signal in central $\mathrm{Pb}-\mathrm{Pb}$ collisions (currently it can be reconstructed only in $\mathrm{pp}$ and $\mathrm{p}-\mathrm{Pb}$ collisions). In Fig. 2 the expected performance after the LS2 for the nuclear modification factor of the $\Lambda_{\mathrm{c}}$ and the double ratio $\left(\Lambda_{\mathrm{c}} / \mathrm{D}\right)_{\mathrm{Pb}-\mathrm{Pb}} /\left(\Lambda_{\mathrm{c}} / \mathrm{D}\right)_{\mathrm{pp}}$ are shown in the left and right hand panels, respectively. In the right-hand panel the double ratio is compared to theoretical predictions: the achievable precision will allow to distinguish among several models providing a better understanding of the hadronization mechanisms.

The performance for the elliptic flow measurements for several heavy-flavour hadrons is shown in Fig. 3. In the left-hand panel the expected performance for prompt $\mathrm{D}^{0}, \mathrm{D}_{\mathrm{s}}$ and $\Lambda_{\mathrm{c}}$ are shown for semi-central $\mathrm{Pb}-\mathrm{Pb}$ collisions. In the right-hand panel of the same figure the elliptic flow of non-prompt $\mathrm{D}^{0}$ and non-prompt $\mathrm{J} / \psi$ are shown for semi-central $\mathrm{Pb}-\mathrm{Pb}$ collisions. The possibility to measure the elliptic flow for several heavy-flavour hadron species, as well as the precision achievable for these measurements will enable the study of $v_{2}$ of charm and beauty hadrons separately and over a broad $p_{\mathrm{T}}$ interval.

\section{Conclusion}

The simulation studies carried out to assess the physics performance of the upgraded ALICE detector show that after the LS2 the nuclear modification factor and elliptic flow measurements will be available for several heavy-flavour hadrons at very low transverse momentum with high precision. These measurements will allow to study the mass-dependence for both $R_{\mathrm{AA}}$ and $v_{2}$. The comparison with theoretical calculations is expected to allow the estimation of the transport coefficients of the QGP for charm and beauty quarks. 
Furthermore new observables will become available, including the production of heavy-flavour baryons, heavy-flavour correlations and heavy-flavour production in association with jets.

\section{References}

[1] M. Gyulassy and M. Plumer, Phys. Lett. B 243, 432 (1990).

[2] M.H. Thoma and M. Gyulassy, Nucl. Phys. B 351, 491 (1991).

[3] Y. L. Dokshitzer and D. E. Kharzeev, Phys. Lett. B 519, 199 (2001).

[4] J. Rafelski and B. Müller, Phys. Rev. Lett. 48, 1066 (1982).

[5] M. He, R. J. Fries and R. Rapp, Phys. Rev. Lett. 110, 112301 (2013).

[6] K. Aamodt et al. (ALICE Collaboration), JINST 3, S088002 (2008).

[7] ALICE Collaboration, Letter of Intent for the Upgrade of the ALICE Experiment, CERNLHCC2012-012, LHCC-I-022.

[8] ALICE Collaboration, Technical Design Report of the ITS Upgrade, CERN-LHCC-2013024/ALICE-TDR-017. 